\title{
Carlson Type Inequalities
}

SORINA BARZA ${ }^{a}$, JOSIP PEČARIĆ ${ }^{b}$ and LARS-ERIK PERSSON ${ }^{a * *}$

a Department of Mathematics, Luleå University, S-97187 Luleå, Sweden;

b Faculty of Textile Technology, University of Zagreb, Pierottijeva 6, 41000 Zagreb, Croatia

(Received 12 February 1997)

A scale of Carlson type inequalities are proved and the best constants are found. Some multidimensional versions of these inequalities are also proved and it is pointed out that also a well-known inequality by Beurling-Kjellberg is included as an endpoint case.

Keywords: Inequalities; Sharp constants; Carlson's inequality; Kjellberg's inequality; Multidimensional inequalities

AMS Subject Classification (1991): 26D15

\section{INTRODUCTION}

First we consider sequences $\left\{a_{n}\right\}, n=1,2, \ldots$ of nonnegative numbers and functions $f$ on $[0, \infty)$. In 1934, Carlson [5] proved that the somewhat curious inequalities

$$
\sum_{n=1}^{\infty} a_{n} \leq \sqrt{\pi}\left(\sum_{n=1}^{\infty} a_{n}^{2}\right)^{1 / 4}\left(\sum_{n=1}^{\infty} n^{2} a_{n}^{2}\right)^{1 / 4}
$$

and

$$
\int_{0}^{\infty}|f(x)| \mathrm{d} x \leq \sqrt{\pi}\left(\int_{0}^{\infty} f^{2}(x) \mathrm{d} x\right)^{1 / 4}\left(\int_{0}^{\infty} x^{2} f^{2}(x) \mathrm{d} x\right)^{1 / 4}
$$

hold and $C=\sqrt{\pi}$ is the best possible constant in both cases.

\footnotetext{
* Corresponding author.
} 
In 1936 Hardy [7] presented two elementary proofs of (1). In particular he observed that (1) in fact follows even from Schwarz inequality $\sum x_{n} y_{n} \leq\left(\sum x_{n}^{2}\right)^{1 / 2}\left(\sum y_{n}^{2}\right)^{1 / 2}$ applied to the sequences $x_{n}=a_{n}\left(\alpha+\beta n^{2}\right)^{1 / 2}$ and $y_{n}=\left(\alpha+\beta n^{2}\right)^{1 / 2}$. Here we only remark that (1) and its generalizations have sense, e.g. in some moment problems (see [8]), in the theory of interpolation (see $[6,9,11]$ ) and optimal reconstruction of a sampling signal (see [4]). Moreover, (1) can be improved in the following way:

$$
\sum_{n=1}^{\infty} a_{n} \leq \sqrt{\pi}\left(\sum_{n=1}^{\infty} a_{n}^{2}\right)^{1 / 4}\left(\sum_{n=1}^{\infty}\left(n-\frac{1}{2}\right)^{2} a_{n}^{2}\right)^{1 / 4}
$$

This fact was probably first noticed by Landau and a proof can be found in [10].

It is now natural to ask ourselves whether the analogous integral inequality

$$
\int_{0}^{\infty} f(x) \mathrm{d} x \leq C\left(\int_{0}^{\infty} f^{2}(x) \mathrm{d} x\right)^{1 / 4}\left(\int_{0}^{\infty}\left(x-\frac{1}{2}\right)^{2} f^{2}(x) \mathrm{d} x\right)^{1 / 4}
$$

holds with $C=\sqrt{\pi}$ ? The answer is negative according to a special case of Theorem 2.1 of this paper (in fact (3) holds with $C=\sqrt{2 \pi}$ ).

In order to clarify ideas and motivate our results in this paper we state and prove our announced Theorem 2.1 in Section 2. In Section 3 we state and prove some generalizations (including some multidimensional ones) of Theorem 2.1. We also point out that a well-known inequality of Beurling-Kjellberg [8, p. 17] is included as one endpoint case. Section 4 is reserved for some concluding remarks and results together with a short bibliography of the actual swedish mathematician Fritz Carlson.

\section{AN INTEGRAL CARLSON TYPE INEQUALITY}

In this section we let $f$ denote a Lebesgue measurable nonnegative function on $[0, \infty)$. 
THEOREM 2.1 If $a>0$, then

$$
\int_{0}^{\infty} f(x) \mathrm{d} x \leq \sqrt{2 \pi}\left(\int_{0}^{\infty} f^{2}(x) \mathrm{d} x\right)^{1 / 4}\left(\int_{0}^{\infty}(x-a)^{2} f^{2}(x) \mathrm{d} x\right)^{1 / 4} .
$$

The inequality is sharp.

Proof Let $\alpha, \beta>0$ and let $S=\int_{0}^{\infty} f^{2}(x) \mathrm{d} x$ and $T=\int_{0}^{\infty}(x-a)^{2}$ $f^{2}(x) \mathrm{d} x$. If $S=\infty$ or $T=\infty$, then (4) holds trivially so we may without loss of generality assume that $S<\infty$ and $T<\infty$. By using the Schwarz inequality and elementary calculations we find that

$$
\begin{aligned}
\left(\int_{0}^{\infty} f(x) \mathrm{d} x\right)^{2} & =\left(\int_{0}^{\infty} f(x) \sqrt{\alpha+\beta(x-a)^{2}} \frac{1}{\sqrt{\alpha+\beta(x-a)^{2}}} \mathrm{~d} x\right)^{2} \\
& \leq \int_{0}^{\infty} f^{2}(x)\left(\alpha+\beta(x-a)^{2}\right) \mathrm{d} x \int_{0}^{\infty} \frac{1}{\alpha+\beta(x-a)^{2}} \mathrm{~d} x \\
& =\left.\frac{1}{\sqrt{\alpha \beta}}(\alpha S+\beta T) \arctan \left((x-a) \sqrt{\frac{\beta}{\alpha}}\right)\right|_{0} ^{\infty} \\
& =\left(\frac{\pi}{2}+\arctan a \sqrt{\frac{\beta}{\alpha}}\right)\left(S \sqrt{\frac{\alpha}{\beta}}+T \sqrt{\frac{\beta}{\alpha}}\right) .
\end{aligned}
$$

By choosing $\alpha=T$ and $\beta=S$ we obtain that

$$
\begin{aligned}
\left(\int_{0}^{\infty} f(x) \mathrm{d} x\right)^{2} & \leq 2\left(\frac{\pi}{2}+\arctan a \sqrt{\frac{S}{T}}\right) \sqrt{S T} \\
& =\left(\pi+2 \arctan a \sqrt{\frac{S}{T}}\right) \sqrt{S T} \\
& <2 \pi \sqrt{S T}=2 \pi\left(\int_{0}^{\infty} f^{2}(x) \mathrm{d} x \int_{0}^{\infty}(x-a)^{2} f^{2}(x) \mathrm{d} x\right)^{1 / 2}
\end{aligned}
$$

and (4) is proved. To get the best constant in the inequality (4) it is natural to study the behaviour of the following functions:

$$
f_{\gamma}(x)=\frac{1}{1+\gamma^{2}(x-a)^{2}}, \quad \gamma>0 .
$$


The corresponding numbers $S$ and $T$ for these functions are

$$
S=S_{\gamma}=\int_{0}^{\infty} f_{\gamma}^{2}(x) \mathrm{d} x=\frac{1}{2 \gamma}\left(\frac{\pi}{2}+\arctan \gamma a+\frac{\gamma a}{1+\gamma^{2} a^{2}}\right)
$$

and

$$
T=T_{\gamma}=\int_{0}^{\infty}(x-a)^{2} f_{\gamma}^{2}(x) \mathrm{d} x=\frac{1}{2 \gamma^{3}}\left(\frac{\pi}{2}+\arctan \gamma a-\frac{\gamma a}{1+\gamma^{2} a^{2}}\right) .
$$

Therefore

$$
\lim _{\gamma \rightarrow \infty} \frac{\left(\int_{0}^{\infty} f_{\gamma}(x) \mathrm{d} x\right)^{4}}{\int_{0}^{\infty} f_{\gamma}^{2}(x) \mathrm{d} x \int_{0}^{\infty}(x-a)^{2} f_{\gamma}^{2}(x) \mathrm{d} x}=4 \pi^{2}
$$

and this means in particular that the best constant in (4) is $\sqrt{2 \pi}$.

Remark 2.2 Our proof above shows that (4) is a strict inequality for every nontrivial function $f$ such that the integrals on the right-hand side converge.

Remark 2.3 By using (2) and a trivial estimate we have the following complement of (4): If $a \leq 0$, then

$$
\int_{0}^{\infty} f(x) \mathrm{d} x \leq \sqrt{\pi}\left(\int_{0}^{\infty} f^{2}(x) \mathrm{d} x\right)^{1 / 4}\left(\int_{0}^{\infty}(x-a)^{2} f^{2}(x) \mathrm{d} x\right)^{1 / 4} .
$$

The constant $\sqrt{\pi}$ is sharp for $a=0$ but it remains an open question to find the best constant when $a<0$.

Remark 2.4 We recall that Kjellberg (see [8]) stated the following counterpart of (2):

$$
\int_{-\infty}^{\infty} f(x) \mathrm{d} x \leq \sqrt{2 \pi}\left(\int_{-\infty}^{\infty} f^{2}(x) \mathrm{d} x\right)^{1 / 4}\left(\int_{-\infty}^{\infty} x^{2} f^{2}(x) \mathrm{d} x\right)^{1 / 4}
$$

as a reformulation of a previous result by Beurling. We note that (6) follows from (4) by making a variable transformation and letting $a \rightarrow \infty$. Moreover it can be proved that (6) implies (4): Let $g(x)=f(x)$ 
if $x>0$ and $g(x)=0$ if $x \leq 0$. Then

$$
\begin{aligned}
\int_{0}^{\infty} f(x) \mathrm{d} x & =\int_{-\infty}^{\infty} g(x) \mathrm{d} x=\int_{-\infty}^{\infty} g(x+a) \mathrm{d} x \\
& \leq \sqrt{2 \pi}\left(\int_{-\infty}^{\infty} g^{2}(x+a) \mathrm{d} x\right)^{1 / 4}\left(\int_{-\infty}^{\infty} x^{2} g^{2}(x+a) \mathrm{d} x\right)^{1 / 4} \\
& =\sqrt{2 \pi}\left(\int_{-\infty}^{\infty} g^{2}(x) \mathrm{d} x\right)^{1 / 4}\left(\int_{-\infty}^{\infty}(x-a)^{2} g^{2}(x) \mathrm{d} x\right)^{1 / 4} \\
& =\sqrt{2 \pi}\left(\int_{0}^{\infty} f^{2}(x) \mathrm{d} x\right)^{1 / 4}\left(\int_{0}^{\infty}(x-a)^{2} f^{2}(x) \mathrm{d} x\right)^{1 / 4}
\end{aligned}
$$

This means that inequality (4) in fact is equivalent with its limiting inequality (6).

\section{SOME GENERALIZATIONS OF THEOREM 2.1}

For later purposes but also of independent interest we begin by stating the following slight generalizations of the inequalities (4) and (5).

THEOREM 3.1 Let $\gamma>1, \gamma^{\prime}=\gamma /(\gamma-1)$ and let $f$ be a measurable function on $\mathbb{R}_{+}$. Then

$$
\int_{0}^{\infty}|f(x)| \mathrm{d} x \leq C\left(\int_{0}^{\infty} f^{2}(x) \mathrm{d} x\right)^{1 / 2 \gamma^{\prime}}\left(\int_{0}^{\infty}|x-a|^{\gamma} f^{2}(x) \mathrm{d} x\right)^{1 / 2 \gamma},
$$

where

$$
C=C_{\gamma, m}=\left[2^{m}(\gamma-1)^{-1 / \gamma^{\prime}} \frac{\pi}{\sin (\pi / \gamma)}\right]^{1 / 2}
$$

with $m=1$ if $a>0$ and $m=0$ if $a \leq 0$.

Proof Let $\alpha, \beta>0$ and let $S=\int_{0}^{\infty} f^{2}(x) \mathrm{d} x$ and $T=\int_{0}^{\infty}|x-a|^{\gamma}$ $f^{2}(x) \mathrm{d} x$. As before we may without loss of generality assume that 
$S<\infty$ and $T<\infty$. By using the Schwarz inequality,

$$
\begin{aligned}
\left(\int_{0}^{\infty} f(x) \mathrm{d} x\right)^{2} & =\left(\int_{0}^{\infty} f(x) \sqrt{\alpha+\beta|x-a|^{\gamma}} \frac{1}{\sqrt{\alpha+\beta|x-a|^{\gamma}}} \mathrm{d} x\right)^{2} \\
& \leq \int_{0}^{\infty} f^{2}(x)\left(\alpha+\beta|x-a|^{\gamma}\right) \mathrm{d} x \int_{0}^{\infty} \frac{\mathrm{d} x}{\alpha+\beta|x-a|^{\gamma}} \\
& =(\alpha S+\beta T) \int_{0}^{\infty} \frac{\mathrm{d} x}{\alpha\left(1+(\beta / \alpha)|x-a|^{\gamma}\right)} \\
& =\left(S+\frac{\beta}{\alpha} T\right)\left(\frac{\alpha}{\beta}\right)^{1 / \gamma} I,
\end{aligned}
$$

where

$$
I=\int_{-a(\alpha / \beta)^{1 / \gamma}}^{\infty} \frac{\mathrm{d} x}{1+|x|^{\gamma}}
$$

when $a>0$ and we conclude that

$$
I \leq 2^{m} \int_{0}^{\infty} \frac{\mathrm{d} x}{1+x^{\gamma}}=2^{m} \frac{\pi}{\gamma \sin (\pi / \gamma)} .
$$

We have still freedom to choose $\alpha$ and $\beta$ in (8) and by minimizing we find that the optimal choice is $\alpha / \beta=(\gamma-1)(T / S)$. Therefore, by (8) and (9), it yields that

$$
\begin{aligned}
\left(\int_{0}^{\infty}|f(x)| \mathrm{d} x\right)^{2} & \leq\left(S+\frac{S}{T} T \frac{1}{\gamma-1}\right)\left((\gamma-1) \frac{T}{S}\right)^{1 / \gamma} 2^{m} \int_{0}^{\infty} \frac{\mathrm{d} x}{1+x^{\gamma}} \\
& =S \frac{\gamma}{\gamma-1}\left(\frac{T}{S}\right)^{1 / \gamma}(\gamma-1)^{1 / \gamma} 2^{m} \frac{\pi}{\gamma \sin (\pi / \gamma)} \\
& =S^{1 / \gamma^{\prime}} T^{1 / \gamma} C^{2} .
\end{aligned}
$$

Remark 3.2 For the case $\gamma=2$ we have again the inequalities (4) and (5).

Theorem 3.1 can be extended to higher dimensions and for the reader's convenience we give the details for dimension $n=2$. 
THEOREM 3.3 Let $f$ be a measurable function on $\mathbb{R}_{+}^{2}$ and let $a_{1}, a_{2}, a_{3}$, $\gamma_{1}, \gamma_{2}$ and $\gamma_{3}$ be real numbers, where $\gamma_{1}, \gamma_{2}, \gamma_{3}>1$. Then

$$
\begin{aligned}
& \int_{0}^{\infty} \int_{0}^{\infty}|f(x, y)| \mathrm{d} x \mathrm{~d} y \\
& \leq C\left(\int_{0}^{\infty} \int_{0}^{\infty} f^{2}(x, y) \mathrm{d} x \mathrm{~d} y\right)^{1 / 2 \gamma_{3}^{\prime} \gamma_{1}^{\prime}} \\
& \quad \times\left(\int_{0}^{\infty} \int_{0}^{\infty}\left|x-a_{1}\right|^{\gamma_{1}} f^{2}(x, y) \mathrm{d} x \mathrm{~d} y\right)^{1 / 2 \gamma_{3}^{\prime} \gamma_{1}} \\
& \quad \times\left(\int_{0}^{\infty} \int_{0}^{\infty}\left|y-a_{3}\right|^{\gamma_{3}} f^{2}(x, y) \mathrm{d} x \mathrm{~d} y\right)^{1 / 2 \gamma_{3} \gamma_{2}^{\prime}} \\
& \quad \times\left(\int_{0}^{\infty} \int_{0}^{\infty}\left|x-a_{2}\right|^{\gamma_{2}}\left|y-a_{3}\right|^{\gamma_{3}} f^{2}(x, y) \mathrm{d} x \mathrm{~d} y\right)^{1 / 2 \gamma_{2} \gamma_{3}},
\end{aligned}
$$

where

$$
C=C_{\gamma_{1}, a_{1}}^{1 / \gamma_{3}^{\prime}} C_{\gamma_{2}, a_{2}}^{1 / \gamma_{3}} C_{\gamma_{3}, a_{3}}
$$

and

$$
C_{\gamma_{i}, a_{i}}=\left[2^{m_{i}}\left(\gamma_{i}-1\right)^{-1 / \gamma_{i}^{\prime}} \frac{\pi}{\sin \left(\pi / \gamma_{i}\right)}\right]^{1 / 2}
$$

with $m_{i}=1$ if $a_{i}>0$ and $m_{i}=0$ if $a_{i} \leq 0, i=1,2,3$.

Proof First we use Theorem 3.1 with $f(x)$ replaced by $g(y)=$ $\int_{0}^{\infty}|f(x, y)| \mathrm{d} x$ to obtain that

$$
\begin{aligned}
& \int_{0}^{\infty} \int_{0}^{\infty}|f(x, y)| \mathrm{d} x \mathrm{~d} y \\
& \leq C_{\gamma_{3}, a_{3}}\left(\int_{0}^{\infty}\left(\int_{0}^{\infty}|f(x, y)| \mathrm{d} x\right)^{2} \mathrm{~d} y\right)^{1 / 2 \gamma_{3}^{\prime}} \\
& \quad \times\left(\int_{0}^{\infty}\left|y-a_{3}\right|^{\gamma_{3}}\left(\int_{0}^{\infty}|f(x, y)| \mathrm{d} x\right)^{2} \mathrm{~d} y\right)^{1 / 2 \gamma_{3}} .
\end{aligned}
$$


Next we use Theorem 3.1 again now with $f(x)$ replaced by $f(x, y)$ (for each fixed $y$ ):

$$
\begin{aligned}
\left(\int_{0}^{\infty} \int_{0}^{\infty}|f(x, y)| \mathrm{d} x\right)^{2} \leq & C_{\gamma_{i}, a_{i}}^{2}\left(\int_{0}^{\infty} f^{2}(x, y) \mathrm{d} x\right)^{1 / \gamma_{i}^{\prime}} \\
& \times\left(\int_{0}^{\infty}\left|x-a_{i}\right|^{\gamma_{i}} f^{2}(x, y) \mathrm{d} x\right)^{1 / \gamma_{i}}
\end{aligned}
$$

for $i=1,2$.

By inserting (12) into (11) and using Hölder's inequality twice we obtain (10) and the proof is complete.

COROLlary 3.4 Let $f$ be a measurable function on $\mathbb{R}_{+}^{2}$ and let $a_{1}, a_{2}, a_{3}$ be real numbers. Then

$$
\begin{aligned}
& \int_{0}^{\infty} \int_{0}^{\infty}|f(x, y)| \mathrm{d} x \mathrm{~d} y \\
& \leq C\left(\int_{0}^{\infty} \int_{0}^{\infty} f^{2}(x, y) \mathrm{d} x \mathrm{~d} y \int_{0}^{\infty} \int_{0}^{\infty}\left(x-a_{1}\right)^{2} f^{2}(x, y) \mathrm{d} x \mathrm{~d} y\right. \\
& \times \int_{0}^{\infty} \int_{0}^{\infty}\left(y-a_{3}\right)^{2} f^{2}(x, y) \mathrm{d} x \mathrm{~d} y \\
&\left.\times \int_{0}^{\infty} \int_{0}^{\infty}\left(x-a_{2}\right)^{2}\left(y-a_{3}\right)^{2} f^{2}(x, y) \mathrm{d} x \mathrm{~d} y\right)^{1 / 8},
\end{aligned}
$$

where

$$
\begin{aligned}
& C=2 \pi \quad \text { if } a_{1}, a_{2}, a_{3}>0, \\
& C=2^{3 / 4} \pi \quad \text { if } a_{1} \leq 0 \text { and } a_{2}, a_{3}>0 \text { or } a_{2} \leq 0 \text { and } a_{1}, a_{3}>0, \\
& C=\sqrt{2} \pi \quad \text { if } a_{1}, a_{2} \leq 0 \text { and } a_{3}>0 \text { or } a_{1}, a_{2}>0 \text { and } a_{3} \leq 0, \\
& C=2^{1 / 4} \pi \quad \text { if } a_{1}>0 \text { and } a_{2}, a_{3} \leq 0 \text { or } a_{2}>0 \text { and } a_{1}, a_{3} \leq 0, \\
& C=\pi \quad \text { if } a_{1}, a_{2}, a_{3} \leq 0 .
\end{aligned}
$$

Proof Apply Theorem 3.3 with $\gamma_{1}=\gamma_{2}=\gamma_{3}=2$. 
Example 3.5 Let $f$ be a measurable function on $\mathbb{R}_{+}^{2}$. Then we have:

(a) [Beurling-Kjellberg type]:

$$
\begin{aligned}
& \int_{-\infty}^{\infty} \int_{-\infty}^{\infty}|f(x, y)| \mathrm{d} x \mathrm{~d} y \\
& \leq 2 \pi\left(\int_{-\infty}^{\infty} \int_{-\infty}^{\infty} f^{2}(x, y) \mathrm{d} x \mathrm{~d} y \int_{-\infty}^{\infty} \int_{-\infty}^{\infty} x^{2} f^{2}(x, y) \mathrm{d} x \mathrm{~d} y\right. \\
&\left.\times \int_{-\infty}^{\infty} \int_{-\infty}^{\infty} y^{2} f^{2}(x, y) \mathrm{d} x \mathrm{~d} y \int_{-\infty}^{\infty} \int_{-\infty}^{\infty} x^{2} y^{2} f^{2}(x, y) \mathrm{d} x \mathrm{~d} y\right)^{1 / 8} .
\end{aligned}
$$

(b) [“intermediate" inequalities]:

$$
\begin{aligned}
\int_{0}^{\infty} & \int_{-\infty}^{\infty}|f(x, y)| \mathrm{d} x \mathrm{~d} y \\
\leq & \sqrt{2} \pi\left(\int_{0}^{\infty} \int_{-\infty}^{\infty} f^{2}(x, y) \mathrm{d} x \mathrm{~d} y \int_{0}^{\infty} \int_{-\infty}^{\infty} x^{2} f^{2}(x, y) \mathrm{d} x \mathrm{~d} y\right. \\
& \left.\times \int_{0}^{\infty} \int_{-\infty}^{\infty} y^{2} f^{2}(x, y) \mathrm{d} x \mathrm{~d} y \int_{0}^{\infty} \int_{-\infty}^{\infty} x^{2} y^{2} f^{2}(x, y) \mathrm{d} x \mathrm{~d} y\right)^{1 / 8} .
\end{aligned}
$$

(c) [Carlson type]:

$$
\begin{aligned}
& \int_{0}^{\infty} \int_{0}^{\infty}|f(x, y)| \mathrm{d} x \mathrm{~d} y \\
& \leq \pi\left(\int_{0}^{\infty} \int_{0}^{\infty} f^{2}(x, y) \mathrm{d} x \mathrm{~d} y \int_{0}^{\infty} \int_{0}^{\infty} x^{2} f^{2}(x, y) \mathrm{d} x \mathrm{~d} y\right. \\
&\left.\quad \times \int_{0}^{\infty} \int_{0}^{\infty} y^{2} f^{2}(x, y) \mathrm{d} x \mathrm{~d} y \int_{0}^{\infty} \int_{0}^{\infty} x^{2} y^{2} f^{2}(x, y) \mathrm{d} x \mathrm{~d} y\right)^{1 / 8}
\end{aligned}
$$

By considering our proof of Theorem 3.3 and using induction it is obvious that we can state and prove an $n$-dimensional version of Theorem 3.3 but here we only state the following $n$-dimensional version of Theorem 2.1 and Corollary 3.4.

THEOREM 3.6 Let $f$ be a real-valued measurable function on $\mathbb{R}_{+}^{n}$, and $A_{n}=\left\{\left\{b_{i}\right\}_{1}^{n}, b_{i}=1\right.$ or $\left.b_{i}=0, i=1,2, \ldots, n\right\}$.

(a) If $a_{i}>0, i=1,2, \ldots, n$, then

$$
\int_{\mathbb{R}_{+}^{n}}|f(x)| \mathrm{d} x \leq(2 \pi)^{n / 2}\left(\prod_{A_{n}} \int_{\mathbb{R}_{+}^{n}} \prod_{1}^{n}\left(x_{i}-a_{i}\right)^{2 b_{i}} f^{2}(x) \mathrm{d} x\right)^{1 / 2^{n+1}} .
$$


(b) If $a_{i} \leq 0, i=1,2, \ldots, n$, then

$$
\int_{\mathbb{R}_{+}^{n}}|f(x)| \mathrm{d} x \leq \pi^{n / 2}\left(\prod_{A_{n}} \int_{\mathbb{R}_{+}^{n}} \prod_{1}^{n}\left(x_{i}-a_{i}\right)^{2 b_{i}} f^{2}(x) \mathrm{d} x\right)^{1 / 2^{n+1}} .
$$

Remark 3.7 By using (a), letting $a_{1}=a_{2}=\cdots=a_{n}=a>0$, making a variable transformation and letting $a \rightarrow \infty$ we obtain the BeurlingKjellberg inequality [8, page 17]:

$$
\int_{\mathbb{R}_{+}^{n}}|f(x)| \mathrm{d} x \leq(2 \pi)^{n / 2}\left(\prod_{A_{n}} \int_{\mathbb{R}_{+}^{n}} \prod_{1}^{n} x_{i}^{2 b_{i}} f^{2}(x) \mathrm{d} x\right)^{1 / 2^{n+1}} .
$$

\section{CONCLUDING REMARKS AND RESULTS}

Proposition 4.1 Let $f_{1}, f_{2}, \ldots, f_{n_{0}}:[0, \infty) \rightarrow[0, \infty)$ be Lebesgue measurable functions $\left(n_{0} \in \mathbb{N}\right)$, let $p_{k}>1, k=1,2, \ldots, n_{0}$, where $\sum_{k=1}^{n_{0}}\left(1 / p_{k}\right)=1$ and let $\gamma>1$. If $a_{k}>0, k=1,2, \ldots, m_{0} \leq n_{0}$ and $a_{k} \leq 0, k=m_{0}+1, \ldots, n_{0}$, then

$$
\begin{aligned}
\int_{0}^{\infty} \prod_{k=1}^{n_{0}} f_{k}(x) \mathrm{d} x \leq & C \prod_{k=1}^{n_{0}}\left(\int_{0}^{\infty} f_{k}^{2 p_{k}}(x) \mathrm{d} x\right)^{\left(1 / 2 p_{k}\right)(1-1 / \gamma)} \\
& \times\left(\int_{0}^{\infty}\left(x-a_{k}\right)^{\gamma} f_{k}^{2 p_{k}}(x) \mathrm{d} x\right)^{1 / 2 p_{k} \gamma}
\end{aligned}
$$

where

$$
C^{2}=(2)^{\sum_{k=1}^{m_{0}}\left(1 / p_{k}\right)}(\gamma-1)^{-1 / \gamma^{\prime}} \frac{\pi}{\sin (\pi / \gamma)}
$$

Proof By applying Theorem 3.1 we find that

$$
\left(\int_{0}^{\infty} g(x) \mathrm{d} x\right)^{2} \leq 2^{m} C_{0}\left(\int_{0}^{\infty} g^{2}(x) \mathrm{d} x\right)^{1-1 / \gamma}\left(\int_{0}^{\infty}\left(x-a_{k}\right)^{\gamma} g^{2}(x) \mathrm{d} x\right)^{1 / \gamma}
$$

where $C_{0}=(\gamma-1)^{-1 / \gamma^{\prime}} \pi / \sin (\pi / \gamma), m=1$ if $a_{k}>0$ and $m=0$ if $a_{k} \leq 0$. 
Moreover, by using the Hölder inequality, we obtain

$$
\begin{aligned}
\left(\int_{0}^{\infty} \prod_{k=1}^{n_{0}} f_{k}(x) \mathrm{d} x\right)^{2} \leq & \prod_{k=1}^{n_{0}}\left(\int_{0}^{\infty} f_{k}^{p_{k}}(x) \mathrm{d} x\right)^{2 / p_{k}} \\
\leq & 2^{\sum_{k=1}^{m_{0}}\left(1 / p_{k}\right)} C_{0} \prod_{k=1}^{n_{0}}\left(\int_{0}^{\infty} f_{k}^{2 p_{k}}(x) \mathrm{d} x\right)^{\left(1 / p_{k}\right)(1-1 / \gamma)} \\
& \times\left(\int_{0}^{\infty}\left(x-a_{k}\right)^{\gamma} f_{k}^{2 p_{k}}(x) \mathrm{d} x\right)^{1 / p_{k} \gamma}
\end{aligned}
$$

and the proof follows.

The special case when $p_{k}=n_{0}, k=1,2, \ldots, n_{0}$ and $\gamma=2$ is of special interest.

Corollary 4.2 Let $f_{1}, f_{2}, \ldots, f_{n_{0}}:[0, \infty) \rightarrow[0, \infty)$ be Lebesgue measurable functions $\left(n_{0} \in \mathbb{N}\right)$. If $a_{k}>0, k=1,2, \ldots, m_{0} \leq n_{0}$ and $a_{k} \leq 0$, $k=m_{0}+1, \ldots, n_{0}$, then

$$
\begin{aligned}
& \int_{0}^{\infty} \prod_{k=1}^{n} f_{k}(x) \mathrm{d} x \\
& \quad \leq(\sqrt{2})^{m_{0} / n_{0}} \sqrt{\pi} \prod_{k=1}^{n_{0}}\left(\int_{0}^{\infty} f_{k}^{2 n_{0}}(x) \mathrm{d} x \int_{0}^{\infty}\left(x-a_{k}\right)^{2} f_{k}^{2 n_{0}}(x) \mathrm{d} x\right)^{1 / 4 n_{0}} .
\end{aligned}
$$

Example 4.3 For $n_{0}=2, a_{1}=a, a_{2}=b, f_{1}=f, f_{2}=g$ Corollary 4.2 reads:

(a) If $a>0, b>0$, then

$$
\begin{aligned}
\int_{0}^{\infty} f(x) g(x) \mathrm{d} x \\
\leq \sqrt{2 \pi}\left(\int_{0}^{\infty} f^{4}(x) \mathrm{d} x \int_{0}^{\infty} g^{4}(x) \mathrm{d} x\right. \\
\left.\quad \times \int_{0}^{\infty}(x-a)^{2} f^{4}(x) \mathrm{d} x \int_{0}^{\infty}(x-b)^{2} g^{4}(x) \mathrm{d} x\right)^{1 / 8} .
\end{aligned}
$$


(b) If $a>0, b \leq 0$ or $a \leq 0, b>0$, then

$$
\begin{aligned}
\int_{0}^{\infty} f(x) g(x) \mathrm{d} x \\
\leq \sqrt[4]{2} \sqrt{\pi}\left(\int_{0}^{\infty} f^{4}(x) \mathrm{d} x \int_{0}^{\infty} g^{4}(x) \mathrm{d} x\right. \\
\left.\quad \times \int_{0}^{\infty}(x-a)^{2} f^{4}(x) \mathrm{d} x \int_{0}^{\infty}(x-b)^{2} g^{4}(x) \mathrm{d} x\right)^{1 / 8} .
\end{aligned}
$$

(c) If $a<0, b<0$, then

$$
\begin{aligned}
& \int_{0}^{\infty} f(x) g(x) \mathrm{d} x \\
& \leq \sqrt{\pi}\left(\int_{0}^{\infty} f^{4}(x) \mathrm{d} x\right. \\
&\left.\quad \times \int_{0}^{\infty} g^{4}(x) \mathrm{d} x \int_{0}^{\infty}(x-a)^{2} f^{4}(x) \mathrm{d} x \int_{0}^{\infty}(x-b)^{2} g^{4}(x) \mathrm{d} x\right)^{1 / 8} .
\end{aligned}
$$

Remark 4.4 Proposition 4.1 may obviously be regarded as a formal generalization of Theorem 3.1. By using the same technique we can obtain a similar generalization of Theorems 3.3 and 3.6.

Remark 4.5 The Hardy idea presented in our introduction was very important for our proof of Theorem 3.1. This idea of proof is completely different from the original proof of Carlson who in fact remarked that (1) does not follow from Hölder's inequality in the following way:

$$
\begin{aligned}
\sum_{n=1}^{\infty} a_{n} & \leq\left(\sum_{n=1}^{\infty} a_{n}^{2}\right)^{1 / 4}\left(\sum_{n=1}^{\infty} n^{2 h} a_{n}^{2}\right)^{1 / 4}\left(\sum_{n=1}^{\infty} n^{-h}\right)^{1 / 2} \\
& =C(h)\left(\sum_{n=1}^{\infty} a_{n}^{2}\right)^{1 / 4}\left(\sum_{n=1}^{\infty} n^{2 h} a_{n}^{2}\right)^{1 / 4}
\end{aligned}
$$

because $C(h) \rightarrow \infty$ when $h \rightarrow 1+$.

Remark 4.6 Let $f^{*}$ denote the nonincreasing rearrangement of $f$. Then by applying (2) with $f=f^{*}$ we find that this inequality can be replaced 
by the following formal sharper one

$$
\int_{0}^{\infty}|f(x)| \mathrm{d} x \leq \sqrt{\pi}\left(\int_{0}^{\infty} f^{2}(x) \mathrm{d} x\right)^{1 / 4}\left(\int_{0}^{\infty} x^{2}\left(f^{*}(x)\right)^{2} \mathrm{~d} x\right)^{1 / 4} .
$$

Some related multidimensional integral inequalities of reversed (Hölder) type have recently been proved in [2].

Remark 4.7 A generalization of (1) in a completely other direction was recently done in [9]. This "block type inequality" was the key step to solve a very old question in interpolation theory. See [9] and cf. also [6,11]. Some other generalizations of Carlson's inequalities can be found in the books [1,12], and the references given there.

Remark 4.8 Let $\Omega$ be an arbitrary infinite cone in $\mathbb{R}^{n}$ with the origin as a vertex, i.e.,

$$
\Omega=\left\{x \in \mathbb{R}^{n}: x=\rho \sigma, 0<\rho<\infty, \sigma \in S\right\},
$$

where $S \subseteq S^{n}=\left\{x \in \mathbb{R}^{n}:|x|=1\right\}$ is a measurable set. In a joint work with professor Victor Burenkov we have recently also obtained the following result for the case with homogeneous weights (see [3]): Let $w_{i}$, $i=0,1,2$, be functions defined on the cone $\Omega$. Suppose that $w_{i}$ are positive, measurable and homogeneous ${ }^{\dagger}$ of orders $\alpha_{i} \in \mathbb{R}, i=0,1,2$. Moreover, suppose that

$$
0<p_{0}<p_{1}, p_{2}<\infty, \quad 0<\theta<1,
$$

and put $d_{i}=\alpha_{i}+n / p_{i}, i=0,1,2$. In order that for some $A>0$ and for all functions $f$ measurable on $\Omega$ and satisfying $\left\|f w_{i}\right\|_{L_{p_{i}}(\Omega)}<\infty$, $i=1,2$,

$$
\left\|f w_{0}\right\|_{L_{p_{0}}(\Omega)} \leq A\left\|f w_{1}\right\|_{L_{p_{1}}(\Omega)}^{\theta}\left\|f w_{2}\right\|_{L_{p_{2}}(\Omega)}^{1-\theta}
$$

it is necessary and sufficient that

$$
d_{0}=\theta d_{1}+(1-\theta) d_{2}, \quad d_{1} \neq d_{2},
$$

\footnotetext{
${ }^{\dagger}$ That is, for each $x \in \Omega$ and $\varepsilon>0, w_{i}(\varepsilon x)=\varepsilon^{\alpha_{i}} w_{i}(x)$.
} 
and

$$
\left\|\frac{w_{0}}{w_{1}^{\theta} w_{2}^{1-\theta}}\right\|_{L_{q}(S)}<\infty
$$

where

$$
\frac{1}{q}=\frac{1}{p_{0}}-\frac{\theta}{p_{1}}-\frac{1-\theta}{p_{2}} .
$$

The proof can be found in [3], where also the best constant $A$ is explicitly calculated and all cases of equality are pointed out.

This result may be regarded as another generalization of the inequalities (2) and (4).

Final note about Fritz Carlson (1888-1952) Fritz Carlson was appointed as professor in Stockholm, Sweden, 1920. His main work was focused on the theory of analytic functions. Some of his most wellknown contributions are a theorem connected to the PhragménLindelöf principle, a theorem about the zero points for the $\xi$-function and theorems concerning power series with integer coefficients.

Carlson's original proofs of the inequalities (1) and (2) illustrate his deep knowledge in the theory of analytic functions. It must have been a big surprise for him when Hardy two years later presented his much more elementary proofs (cf. Remark 4.5).

Carlson was a really correct professor with great authority in the swedish tradition of that time. He was one of the editors of the wellknown Swedish Journal Acta Mathematica and for a period the chief of the Mittag-Leffer institute.

\section{Acknowledgement}

We thank Professor Victor Burenkov for some generous advises and suggestions which have improved the final version of the paper.

\section{References}

[1] E.F. Beckenbach and R. Bellman, Inequalities, 2nd edn., Springer-Verlag, BerlinNew York, 1965.

[2] S. Barza, J. Pečarić and L-E. Persson, Reversed Hölder type inequalities for monotone functions of several variables, Math. Nach., 186 (1997), 67-80. 
[3] S. Barza, V. Burenkov, J. Pečarić and L-E. Persson, Sharp multidimensional multiplicative inequalities for weighted $L_{p}$-spaces with homogeneous weights, Math. Ineq. Appl. (to appear).

[4] J. Bergh, An optimal reconstruction of sampled signals, J. Math. Anal. Appl., 115 (1986), 574-577.

[5] F. Carlson, Une inegalité, Ark. Mat. Astr. Fysik, 25B (1934), 1-5.

[6] J. Gustavsson and J. Peetre, Interpolation of Orlicz spaces, Studia Math., 60 (1977), $33-59$.

[7] G.H. Hardy, A note on two inequalities, J. London Math. Soc., 11 (1936), 167-170.

[8] B. Kjellberg, On some inequalities, C.R. Dixième Congrès des Mathematiciens Scandinaves (1946), Jul. Gjellerups Forlag, Copenhagen (1946), 333-340.

[9] N.Ya. Krugljak, L. Maligranda and L.E. Persson, A Carlson type inequality with blocks and interpolation, Studia Math., 104 (1993), 161-180.

[10] V.I. Levin and S.B. Stečkin, Inequalities, Amer. Math. Soc. Transl., 14 (1960), 1-22.

[11] L. Maligranda and L.E. Persson, Inequalities and interpolation, Collect. Math., 44 (1993), 183-201.

[12] D.S. Mitrinović, J.E. Pečarić and A.M. Fink, Inequalities Involving Functions and Their Integrals and Derivatives, Kluwer Acad. Publ., Dordrecht/ Boston/ London (1991). 